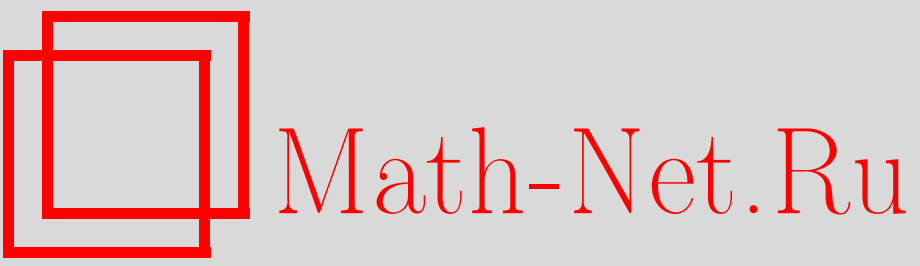

У. А. Розиков, Периодические гиббсовские меры неоднородной модели Изинга на деревьях, УМH, 2001, том 56, выпуск 1, 175-176

DOI: https://doi.org/10.4213/rm371

Использование Общероссийского математического портала Math-Net.Ru подразумевает, что вы прочитали и согласны с пользовательским соглашением

http://www.mathnet.ru/rus/agreement

Параметры загрузки:

IP: 54.224 .187 .69

26 апреля 2023 г., 17:45:52 


\title{
ПЕРИОДИЧЕСКИЕ ГИББСОВСКИЕ МЕРЫ НЕОДНОРОДНОЙ МОДЕЛИ ИЗИНГА НА ДЕРЕВЬЯХ
}

\author{
У. А. Розиков
}

Деревом назьвается связный граф̆, не содержащий циклов. Одним из частных случаев дерева является дерево Кэли, т.е. дерево, из каждой вершины которого выходит ровно $k+1$ ребро (дерево Кэли порядка $k \geqslant 1$ ). На дереве Кэли изучены модели статистической механики, и для них описаны множества периодических гиббсовских мер, а также изучены траектории случайного блуждания в случайной среде [1]-[4].

Для изучения этих задач используется групповое представление дерева Кэли [1], в этом случае задачи формулируются достаточно просто на языке группы, которая представляет дерево Кэли.

Заметим, что деревья Кэли и только они представимы как группа.

Пусть $\Gamma$ - бесконечное дерево, т.е. граф без циклов. Предположим, что из каждой точки $x \in \Gamma$ выходит не менее 1 и не более $k_{\Gamma}<+\infty$ ребер.

Обозначим через $V$ множество вершин и через $L$ множество ребер дерева $\Gamma$.

Для $x, y \in V$ мы будем писать $x \leqslant y$, если $x$ находится на кратчайшем пути, соединяющем $x^{0}$ и $y$; будем писать $x<y$, если $x \leqslant y$ и $x \neq y$. Если $x \leqslant y$ и $|y|=|x|+1$, тогда будем писать $x \rightarrow y$. Точку $x^{0}$ назовем корнем дерева $\Gamma$.

Обозначим

$$
S(x)=\{y \in V: x \rightarrow y\} .
$$

Пусть даны натуральные числа:

$$
\begin{gathered}
\alpha_{1} ; \alpha_{1 i_{1}}, i_{1}=1,2, \ldots, \alpha_{1} ; \alpha_{1 i_{1} i_{2}}, i_{2}=1,2, \ldots, \alpha_{1 i_{1}} ; \\
\ldots ; \alpha_{1 i_{1} i_{2} \ldots i_{n}}, i_{n}=1,2, \ldots, \alpha_{1 i_{1} i_{2} \ldots i_{n-1}} ; \ldots
\end{gathered}
$$

Обозначим через $A$ множество таких чисел. Пусть

$$
\begin{aligned}
K_{A}=\left\{\left(i_{0}, i_{1}, i_{2}, \ldots, i_{n}\right): i_{0}=1 ; i_{1}=1,2, \ldots, \alpha_{1} ;\right. \\
\left.\quad i_{2}=1,2, \ldots, \alpha_{1} i_{1} ; \ldots ; i_{n}=1,2, \ldots, \alpha_{1 i_{1} i_{2} \ldots i_{n-1}} ; n \geqslant 0\right\} .
\end{aligned}
$$

Пусть $B$ - множество всевозможных $A$ и $\Lambda$ - множество всевозможных деревьев $\Gamma$.

ПреДЛОЖЕНИЕ 1. Существует взаимнооднозначное соответствие $F$ между әлементами $\Lambda$ и $B$.

Обозначим через $A(\Gamma)$ множество вида $A$, соответствующее дереву $\Gamma$.

ПредложенИЕ 2. Для любого дерева Г существует взаимнооднозначное соответствие между әлементами множества $K_{A(\Gamma)}$ и множеством вериин $V$ дерева $\Gamma$.

Таким образом, любое дерево Г мы можем представить как множество вида $K_{A(\Gamma)}$.

Пусть $\xi=\left\{\xi_{1}, \xi_{2}, \ldots, \xi_{n}, \ldots\right\}$ - некоторое разбиение множества $K=K_{A(\Gamma)}$ на классы и $h: K \rightarrow \mathbb{R}$ - произвольное отображение.

ОПРЕДЕлЕнИЕ 1. Отображение $h$ назовем $\xi$-периодическим, если $h_{x}=h_{i}$ для любого $x \in$ $\xi_{i}, i=1,2, \ldots$

Пусть $x \in$ Г и ему соответствует $\left(1, i_{1}, i_{2}, \ldots, i_{n}\right) \in K$, тогда, очевидно, $|x|=n$. Пусть $\omega_{x}(j)$ - число $j$, участвующих в записи $x=1, i_{1}, i_{2}, \ldots, i_{|x|}, j \leqslant k_{\Gamma}$. Для любого $B \subseteq N_{\Gamma}=$ $\left\{1,2, \ldots, k_{\Gamma}\right\}$ строим следующее разбиение: $\xi_{B}=\left\{\xi_{1}, \xi_{2}\right\}$, где $\xi_{1}=\left\{x \in K: \sum_{j \in B} \omega_{x}(j)\right.$ четно $\}, \xi_{1}=\left\{x \in K: \sum_{j \in B} \omega_{x}(j)\right.$ нечетно $\}$.

Пусть $K$ - представление дерева $Г$. Обозначим

$W_{n}=\{x \in K:|x|=n\}, \quad V_{n}=\{x \in K:|x| \leqslant n\}, \quad L_{n}=\left\{l=\langle x, y\rangle \in L: x, y \in V_{n}\right\}$.

Гамилттониан модели Изинга (неоднородной) на дереве Г имеет вид:

$$
H(\sigma)=-\sum_{\langle x, y\rangle \in L} J_{x y} \sigma(x) \sigma(y),
$$

где $J_{x y} \in \mathbb{R}_{+}, \sigma(x) \in\{-1,1\}$ для любого $x, y \in K$. 
Положим

$$
\Xi=\left\{h=\left(h_{x}, x \in K\right): h_{x}=\sum_{y \in S(x)} f\left(h_{y} ; \theta_{x y}\right)\right\},
$$

где $f(x ; \theta)=\operatorname{arcth}(\theta \tanh x), \theta_{x y}=\tanh \left(\frac{J_{x y}}{T}\right), T>0$. Известно, что каждому $h \in \Xi$ соответствует, и притом единственная, мера Гиббса $\mu$ на $\Omega=\{-1,1\}^{K}$.

Таким образом, задача описания предельных гиббсовских мер сводится к описанию элементов множества $\Xi$. Описание произвольного элемента $\Xi$ - сложная проблема. В данной работе мы ограничимся описанием $\xi_{N_{\Gamma}}$-периодических элементов $\Xi$.

Для описания $\xi_{N_{\Gamma}}$-периодических гиббсовских мер, т.е. описанию функции $h_{x}, x \in K$, вида:

$$
h_{x}= \begin{cases}h_{1}, & \text { если }|x| \text { четно, } \\ h_{2}, & \text { если }|x| \text { нечетно. }\end{cases}
$$

Для $h_{1}, h_{2}$ в случае $x=1$ получим систему уравнений

$$
\begin{cases}h_{1}=\sum_{i=1}^{\alpha_{1}} f\left(h_{2} ; \theta_{1 ; 1, i}\right), & (*) \\ h_{2}=\sum_{j=1}^{\alpha_{1 i}} f\left(h_{1} ; \theta_{1, i ; 1, i, j}\right) . & (*) .\end{cases}
$$

Пусть выполняется неравенство

$$
\sum_{i=1}^{\alpha_{1}}\left(\theta_{1 ; 1, i} \sum_{j=1}^{\alpha_{1 i}} \theta_{1, i ; 1, i, j}\right) \geqslant 1
$$

Для любого $i=1,2, \ldots, \alpha_{1}$ предположим выполнение следующих равенств:

$$
\sum_{j=1}^{\alpha_{1 i}} f\left(h_{1}^{*} ; \theta_{1, i ; 1, i, j}\right)=\sum_{j=1}^{\alpha_{11}} f\left(h_{1}^{*} ; \theta_{1,1 ; 1,1, j}\right),
$$

где $h_{1}^{*}$ - решение уравнения, полученного при подставлении уравнения $(*)$ в $(*)$.

Таким образом, если вьполняются соотношения (2) и (3), то система уравнений (1) имеет три решения:

Для того чтобы функция

$$
h^{0}=(0 ; 0), \quad h^{ \pm}=\left( \pm h_{1}^{*}, \pm h_{2}^{*}\right), \quad \text { где } h_{2}^{*}=\sum_{j=1}^{\alpha_{11}} f\left(h_{1}^{*} ; \theta_{1,1 ; 1,1, j}\right) .
$$

$$
\pm h_{x}=\left\{\begin{array}{l} 
\pm h_{1}^{*}, \text { если }|x| \text { четно, } \\
\pm h_{2}^{*}, \text { если }|x| \text { нечетно, }
\end{array}\right.
$$

удовлетворяла условию согласования, необходимо и достаточно выполнение условий (условие на параметры $\left.\theta_{x y}\right)$

$$
\left\{\begin{array}{l}
h_{1}^{*}=\sum_{y \in S(x)} f\left(h_{2}^{*} ; \theta_{x y}\right), \\
h_{2}^{*}=\sum_{z \in S(y)} f\left(h_{1}^{*} ; \theta_{y z}\right)
\end{array}\right.
$$

для $x \in K \backslash\{1 ; 1, i\}, i=1,2, \ldots, \alpha$.

Теорема. Если параметры $\theta x y, x, y \in K$, удовлетворяют условиям (2)-(4), тогда для неоднородной модели Изинга на дереве Г с представлением К существуют три $\xi_{N_{\Gamma}}$-периодические мерь.

\section{СПИСОК ЛИТЕРАТУРЫ}

[1] Н. Н. Ганиходжаев // Докл. АН РУз. 1994. № 4. С. 3-5. [2] Н. Н. Ганиходжаев, У. А. Р озиков // ТМФ. 1997. Т. 111. № 1. С. 109-117. [3] У. А. Розиков // ТМФ. 1997. Т. 112. № 1. С. 170-175. [4] Н. Н. Ганиходжаев, У. А. Розиков // Матем. сб. 1999. Т. 190. № 2. C. $31-42$. 